\title{
Bilateral Ovarian Leiomyoma in Postmenopausal Women: A Case Report
}

MK Eken, T Senol, EI Kaygusuz, T Erekul, E Cogendez, R Karakus, A Karateke

\begin{abstract}
Primary leiomyoma of the ovary is very rare tumor and most of which occurs in premenopausal women. Patients are mostly asymptomatic and it is diagnosed incidentally by histological examination of ovarian tissue by the pathologist after the removal the ovary.

We first report case of a bilateral primary ovarian leiomyoma in a 53 years old postmenopausal woman incidentally.
\end{abstract}

Keywords: İncidental, leiomyoma ovarii, Postmenopausal

From: Zeynep Kamil Training and Education Hospital, Obstetric and Gynaecology Deprtment, Opr DR Burhanettin Ustunel Cad No 10, Uskudar, Istanbul, Istanbul Turkey.

Correspondence: Dr MK Eken, Zeynep Kamil Education and Research Hospital Obstetric and Gynaecology Department, Uskudar, Istanbul, Turkey. E-mail: meryemkurek@yahoo.com 


\section{INTRODUCTION}

Primary leiomyoma of the ovary is a very rare tumor, which accounts for $0.5-1 \%$ of all benign ovarian tumors (1). Most of these tumors are usually unilateral and only a few millimeters in diameter. Primary ovarian leiomyoma occurs most commonly in women aged 20-65, with $85 \%$ occurring in premenopausal women (2,3). Most patients are asymptomatic, and the tumors are usually not detected on pelvic examination. Rather, they are diagnosed incidentally by histological examination of ovarian tissue by the pathologist after the removal of the pelvic reproductive organs for other situations (4). Uterus leiomyomas are frequently present at the same time as the ovarian leiomyomas. Approximately $15 \%$ of cases occur in postmenopausal women $(5,6)$. Ovarian leiomyomas probably arise from smooth muscle cells in the ovarian hilar blood vessels. Other possible origins are cells in the ovarian ligament, smooth muscle cells or multipotential cells in the ovarian stroma, undifferentiated germ cells, and cortical smooth muscle metaplasia (7).

We first report a case of a bilateral primary ovarian leiomyoma in a 53 years old menopausal woman.

\section{CASE REPORT}

A 53-year-old gravida 5 para 3 woman was admitted to our hospital with a history of lower abdominal pain and postmenapausal uterine bleeding. Physical examination revealed stage 3 pelvic organ prolapse (POP). Physical and abdominal examination findings were normal. Transvaginal ultrasonography revealed a right adnexial mass $22 \mathrm{~mm}$ x $20 \mathrm{~mm}$ and left adnexial mass $20 \mathrm{~mm} \times 18 \mathrm{~mm}$ in diameter that showed a homogeneously isoechoic pattern. Serum AFP:1.82U/ml, CA125:11.4 U/ml, CA 15-3: 14.8U/ml, CA 19-9:<2U/ml values were within normal limits. İn Doppler ultrasonography investigation the masses wereas solid and well circumscribed, and not pathologic blood flow was detected in both of masses. Due to 
postmenopausal bleeding, an endometrial sampling was made from the patient. The pathological examination of endometrial tissues were reported to be in conformity with the postmenopausal period. Due to POP, a decision was taken to implement abdominal hysterectomy, bilateral salpingo-oophorectomy and abdominal sacrocolpopexy on the patient. In the exploration, the uterus and the tubes were observed to be normal. A cyst, which is approximately $2 \mathrm{~cm}$ in size, was detected in both ovaries. Frozen section analysis of ovarian cysts was reported to be in conformity with the benign ovarian cyst. The pathological examination revealed a $2 \mathrm{~cm}$ bilateral ovarian leiomyoma in both the right and the left ovary (Figure-1).

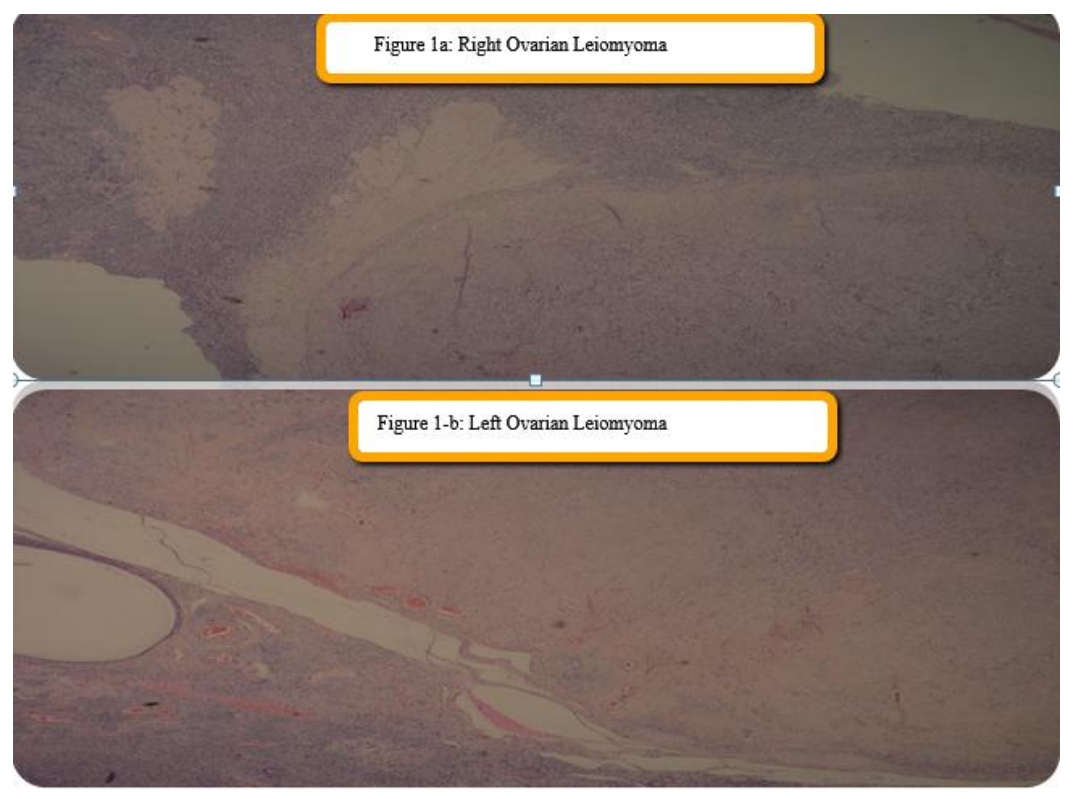

Fig 1-a: Right ovarian Leiomyoma (H\&E X100), Fig 1-b: Left Ovarian Leiomyoma (H\&EX100)

No mitosis, atypia or necrosis were observed in the histological examination. In the immunohistochemical statining, both lesions were detected to be positive through smooth 
muscle actin (Figure-2a), desmine and caldesmon (Figure2-b). The examination of the uterus revelaed a great number of leimyomas, the largest of which was $3 \mathrm{~cm}$ in size.

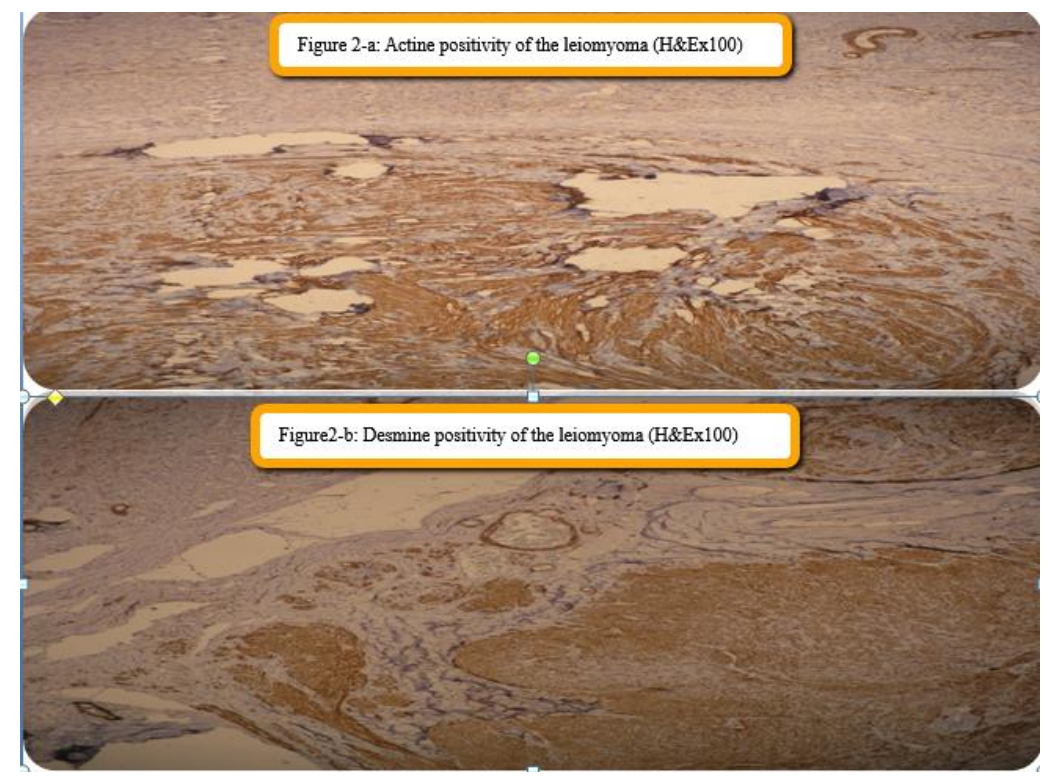

Fig 2-a: Actin Positivity of Leiomyoma (H\&E X100), Fig 2-b: Desmin Positivity of Leiomyoma (H\&E X100)

\section{DISCUSSION}

Ovarian leimyoma was first described by Sangalli in 1862 (8). It is often observed in the postmenopausal period and it is usually smaller than $3 \mathrm{~cm}(9,10)$. There is yet no clear understanding as to the natural course of ovarian leimyoma. The majority of the cases are asymptomatic and incidentally diagnosed. Most of the cases in the relevant literature are unilateral and observed together with uterine leimyomas. Bilateral ovarian leimyoma was first defined in a girl aged 16 by San Marco, et. al. In their cases, the uterus was normal and there was no leimyoma. All the other bilateral cases in relevant literature are in the premenopausal 
period and diagnosed together with uterin leimyomas. Hence, it is considered that the oestrogen effect has a role in their etiologies (5). The fact that there are a great number of uterine leimyomas in our case supports this consideration. There are some studies, alleging that the ovary stems from the blood vessel walls located in the hilus region (8). It has also been stated that leimyoma may result from ovarian ligaments, hilus and multipotent cells in the stroma of the ovary $(8,11,12,13)$. Smooth muscle metaplasia of the theca externa or the stromal cells of the ovarian cortex is also regarded as one of the possible mechanisms (11-12). When ovarian leimyomas stem from above-mentioned tissues within the ovary, they are taken as primary leimyomas; and when they result from the sticking of the pedicled subserosal uterine leimyomas onto the ovarian surface and the loss of its pedicle, they are taken as secondary leimyomas $(2,14)$. In our pathological examination, leimyomas were taken as primary due to the fact that they stem from the ovarian tissue and that they lack leimyoma adhesion over the ovarian exterior surface. In terms of histology, ovarian leimyoma is often diagnosed by being unproblematically distinguished from other fusiform cell tumours thanks to its characteristic histological findings. However, when the tumour is small or largely contains stromal fibrozis, there can be some diagnostic problem due to its findings coinciding with sex-cord cell tumors such as fibroma/thecoma. In terms of immunohistochemistry, exposure of desmine, smooth muscle actin (SMA) and smooth muscle differentiation can resolve the problem in such difficult cases. SMA is negatives in thecomas, but it is strongly observed to be positive in leimyomas. It has been detected that desmine and some thecomas get stained, but that all cells are positively stained in leimyomas. It is thus important to note the amount of stained cells in desmin-positivity. Examination of the ovarian leimyoma in terms of atypia, necrosis and mitosis is significant for the differential diagnosis of leimyoma (15). No mitosis or necrosis was detected in our cases and leimyosarcoma was thus excluded. Our case do not have any clinical finding regarding ovarian cyst in the moment of diagnosis, 
which was incidentally made during the pathological examination of the ovaries. It is hard to obtain a preoperative diagnosis for primary ovarian leimyoma. This tumour, which is often asymptomatic in nature, is detected through pathological examination and immunuhistochemical staining. A literature survey will suffice to show that most case reports are composed of young adults or adolescents with a unilateral tumour (10). The difference of the case from the expected clinical analysis of primary ovarian leimyoma is the fact that it is in the postmenopusal period and has a bilateral tumour. 


\section{REFERENCES}

1. Tomas D, Lenicek T, Tuckar N, et al: Primary ovarian leiomyoma associated with endometriotic cyst presenting with symptoms of acute appendicitis: a case report. Diagn Pathol 2009;4:25.

2. Monk BJ, Nieberg R, Berek JS: Primary leiomyosarcoma of the ovary in a perimenarchal female. Gynecol Oncol 1993; 48:389.

3. Morgante G, Bernabei A, Facchini C, et al: Leiomyoma of the ovary. Case report. Clin Exp Obstet Gynecol 1995; 22:312.

4. Tsalacopoulos G, Tiltman AJ. Leiomyoma of the ovary. S Afr Med J 1981;59:574- 5.

5. Lim S, Jeon H. Bilateral primary ovarian leiomyoma in a young women: case report and literature review. Gynecol Oncol 2004;95:733-735.

6. Kobayashi Y, Murakami R, Sugizaki K, et al. Primary leiomyoma of the ovary: a case report. Eur Radiol 1998;8:1444-1446.

7. Tomas D, Lenicek T, Tuckar N.et al. Primary ovarianleiomyoma associated with endometriotic cyst presenting with symptoms ofacute appendicitis: a case report. Diagn Pathol 2009;4:25.

8. Fallahzadeh H, Dockerty MB, Lee RA. Leiomyoma of the ovary: a report of five cases and review of the literature. Am J Obstet Gynecol 1972;113:394- 8.

9. Matamala MF, Nogales FF, Aneiros J,et al. Leiomyomas of the ovary. Int J Gynecol Pathol 1988;7: 190-6.

10. Wei C, Lilic N, Shorter N, Garrow E. Primary ovarian leiomyoma: a rare cause ofovarian tumor in adolescence. J Pediatr Adolesc Gynecol 2008;21(1):33-6.

11. Lastarria D, Sachdev RK, Babury RA et al. Immunohistochemical analysis for desmin in normal and neoplastic ovarian stromal tissue. Arch Pathol Lab Med 1990;114:5025. 
12. Czernobilsky B, Shezen E, Lifschitz-Mercer B.et al. Alpha smooth muscle actin (alpha-SM actin) in normal human ovaries, in ovarian stromal hyperplasia and in ovarian neoplasm. Virchows Arch B Cell Pathol Incl Mol Pathol 1989;57: 55- 61.

13. Doss BJ, Wanek SM, Jacques SM, et al. Ovarian smooth muscle metaplasia: an uncommon and possibly underrecognized entity. Int J Gynecol Pathol 1999;18:58 -62.

14. Emovon EU, Cahill DJ, Joels LA, et al: Bilateral primary ovarian leiomyoma: a rare cause of ovarian tumour in a young woman. J Obstet Gynecol 1999; 19:676.

15. Tomas D, Lenicek T, Tuckar N, et al. Primary ovarian leiomyoma associated with endometriotic cyst presenting with symptoms ofacute appendicitis: a case report. Diagn Pathol 2009;4:25. 